\title{
Correcting the CIHR course
}

Published at www.cmaj.ca on Sept. 29.

I nstitutional strategic plans are often very much like election campaign platforms - glossy blueprints chock full of marvels that promise everything to everyone and then are systematically set aside to collect bookshelf dust.

Occasionally, though, they actually signal a change in direction, particularly when they're crafted by an agency with an impassioned leader who appears determined to affect change.

The Canadian Institutes of Health Research (CIHR) may be in such a position as President Dr. Alain Beaudet is nothing if not impassioned about aspects of Health Research Roadmap, the granting council's draft strategic plan for 2010-14.

Beaudet bristles at the proposition that the plan is so nebulous as to constitute little more than a broad plea for generous injections of federal cash to essentially maintain the status quo.

On the contrary, he contends. In fact, Beaudet argues that when implemented, the plan will actually prompt major change in the nature of research and the type of research operations that will be supported by CIHR. To wit:

- considerably more support for socalled "translational research," work that actually affects bedside delivery of health care, such as studies comparing the efficacy of diagnostic tests and alternative forms of treatment, particularly drugs;

- more focusing of research on multifaceted, targeted national programs in select areas of national need, such as dementia, Aboriginal health and chronic diseases;

- an expansion of the research umbrella to encompass more players than just those within academe, particularly doctors, nurses and provincial governments.

When it was created in 2000, CIHR vowed to expand the scope of its activities beyond basic, biomedical science

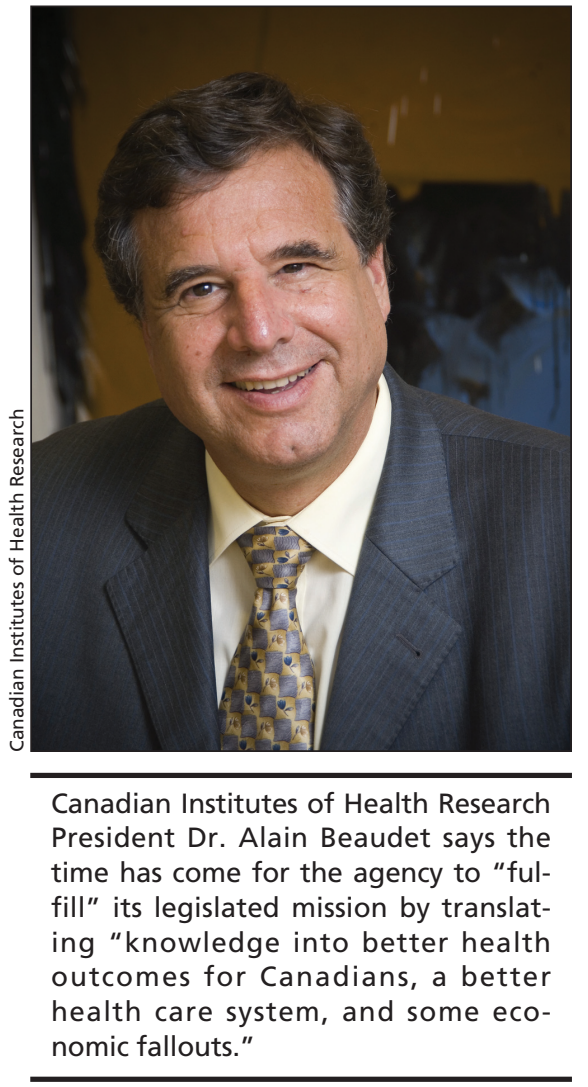

to include more clinical, population health and health services and systems research in exchange for a significant hike in its budget. The agency received the increase; its base budget grew from $\$ 250$ million in fiscal year $1999 / 00$ to $\$ 733.3$ million for fiscal year 2009/10.

But CIHR's foray into health as opposed to biomedical research has been tepid and was fought tooth and nail by the core community that the agency traditionally served, eventually resulting in a significant devolution of financial decision-making authority from its governing council to a committee composed primarily of the scientific directors of its 13 "virtual institutes," (CMAJ 2007;177:605-6).

Yet, Beaudet says that while the agency must continue to fund excellence in basic biomedical research, the time has come to "fulfill the mission of CIHR" as defined in its enabling legislation, and begin to "excel at translating this knowledge into better health outcomes for Canadians, a better health care system and some economic fallouts."

"That is a tall order and it's not an easy thing to do. But that's where I believe we still have some work to do to truly deliver on our mission. That's why we do, indeed, have a lot in here on translation. Translation in terms of economic benefits, commercialization, better and smoother partnerships with the private sector to maximize our efforts, ensuring that we are also poised to respond to the needs of the private sector so that we can actually contribute to enriching our industrial fabric."

"But it's also ensuring that, in the end, the results of research benefit Canadians, benefit our health care system and improve health outcomes. And that's all about not only getting a more efficient bench-to-bedside, but also working hard, once you have evaluated the diagnostics, the new treatments, the new drugs, the new devices. At the bedside, we should be better at translating the results of that evaluation into systematic reviews, guidelines, integration into care, integration into our health care system and, basically, improve our health care system."

To that end, the strategic plan proposes the creation of a Drug Safety and Effectiveness Network to study postmarket safety and effectiveness of drugs, as well as a "strategy for patientoriented care," or what is essentially a plan to enhance national capacity to conduct clinical trials of drugs, treatments and even basic clinical practices, such as the wearing of masks and gloves.

That will require far more coordination of clinical research, more involvement from doctors, nurses and health administrators, as well as the support of the provinces, Beaudet says.

"It's a real challenge but I think we need to face it. We need better organization, getting our act together, working with the provinces, having the provinces tell Ottawa that they need it, 
that they believe in it, they believe that it's absolutely necessary. And yes, we'll need more money, but let's get our act together first. Lots of things, just by being better organized, you know, having national standard operating procedures, national contracts, better ethics review systems for multicentred clinical trials. Just there you've achieved a lot. Then you add the millions and you can start funding clinical trials of the type that we should be funding."

Beaudet also proposes that CIHR adopt a similar sort of rigour and focus in its involvement in strategic and thematic research in areas of national need.

CIHR is already actively involved in organizing an international initiative to collaboratively launch a major investment into the early diagnosis and treatment of Alzheimer disease and other forms of dementia.

Other thematic possibilities for more focused and better-financed strategic programs include:

- Aboriginal health,

- global health problems (specific to less-developed nations),

- chronic diseases,

- emerging threats, such as pandemic (H1N1) 2009, alternatives to medical isotopes in diagnostic testing or hospital-based infections.

Beaudet adds that having made choices for priority areas of investigation, it is equally important that CIHR and Canada put its money where its mouth is. "Not a very large number (of areas). But if you decide to go there, put the means there. To be credible with our international partners also, we've got to put a significant amount of money on the table."

To ensure that CIHR stays firmly on the course set in the draft strategic plan, Beaudet plans to develop and issue annual implementation plans on the strategy, complete with clear performance indicators and accountability measures. "That will also allow us to rectify the course if we're going astray."

The draft plan proposes to synchronize CIHR activity with the federal government's overall science and technology strategy, Mobilizing Science and Technology to Canada's Advantage, as well as the seven subpriority areas of strategic significance identified by the federal Science, Technology and Innovation Committee. Those included four areas in the health and life sciences (regenerative medicine, neuroscience, health in an aging population, and biomedical engineering and medical technologies), as well as one each in environment (water), natural resources and energy, Arctic (climate change adaptation), and information and communications technology (electronic health records).

The strategic plan proposes to achieve that synchronization by focusing future efforts in four directions: "investing in world-class excellence; addressing health and health system research; accelerating the capture of health and economic benefits of health research; and achieving organizational excellence, fostering ethics and demonstrating impact."

Within each of those strategic directions, a number of specifics are proposed. The investing in world-class excellence objective, for example, will include enhanced support for postdoctoral trainees and early career researchers, a review of the peer review system, the inclusion of "potential for impact" as one of the criteria assessing research protocols and some form of new programming to better support breakthrough research because many scientists believe peer reviewers now essentially vote to fund projects that reaffirm the tenets of their own scientific church rather than fund risky, innovative research.

Beaudet surmises that a "special process" for assessing and funding such blue-sky research will be necessary. "It's like an investment portfolio," he says. "You're looking for a balanced portfolio, between the risk and the blue chip."

Among other measures advocated in the plan is the creation of some form of "national health framework," including representation from the general population for setting research priorities. - Wayne Kondro, CMAJ

DOI:10.1503/cmaj.109-3064 